\title{
Central Nervous System Virus Infection in African Children with Cerebral Malaria
}

\author{
Douglas G. Postels, ${ }^{1,4 \star}$ Lawrence Osei-Tutu, ${ }^{2}$ Karl B. Seydel, ${ }^{3,4}$ Qian Xu, ${ }^{5}$ Chenxi Li, ${ }^{5}$ Terrie E. Taylor, ${ }^{3,4}$ Chandy C. John, ${ }^{6}$
} Macpherson Mallewa, ${ }^{7}$ Tom Solomon, ${ }^{8}$ Tsiri Agbenyega, ${ }^{2}$ Daniel Ansong, ${ }^{2}$ Robert O. Opoka, ${ }^{9}$ Lillian M. Khan, ${ }^{10}$ Prashanth S. Ramachandran, ${ }^{11}$ Kristoffer E. Leon, ${ }^{12}$ Joseph L. DeRisi, ${ }^{10,13}$ Charles Langelier, ${ }^{14}$ and Michael R. Wilson ${ }^{11}$

${ }^{1}$ Department of Neurology, Children's National Medical Center, George Washington University, Washington, District of Columbia; ${ }^{2}$ Kwame Nkrumah University of Science and Technology, Kumasi, Ghana; ${ }^{3}$ Department of Osteopathic Medical Specialties, Michigan State University, East

Lansing, Michigan; ${ }^{4}$ Blantyre Malaria Project, University of Malawi College of Medicine, Blantyre, Malawi; ${ }^{5}$ Department of Epidemiology and

Biostatistics, Michigan State University, East Lansing, Michigan; ${ }^{6}$ Ryan White Center for Pediatric Infectious Diseases and Global Health,

Department of Pediatrics, Indiana University School of Medicine, Indianapolis, Indiana; ${ }^{7}$ University of Malawi College of Medicine, Blantyre, Malawi;

8 Institute of Infection and Global Health, University of Liverpool, Liverpool, United Kingdom; ${ }^{9}$ Department of Paediatrics and Child Health,

Makerere University School of Medicine, Kampala, Uganda; ${ }^{10}$ Department of Biochemistry and Biophysics, University of California San Francisco,

San Francisco, California; ${ }^{11}$ Weill Institute for Neurosciences, Department of Neurology, University of California San Francisco, San Francisco,

California; ${ }^{12}$ UCSF School of Medicine, University of California San Francisco, San Francisco, California; ${ }^{13}$ Chan Zuckerburg Biohub, San

Francisco, California; ${ }^{14}$ Division of Infectious Diseases, Department of Medicine, University of California San Francisco, San Francisco, California;

\begin{abstract}
We aimed to identify the contribution of central nervous system (CNS) viral coinfection to illness in African children with retinopathy-negative or retinopathy-positive cerebral malaria (CM). We collected cerebrospinal fluid (CSF) from 272 children with retinopathy-negative or retinopathy-positive CM and selected CSF from 111 of these children (38 retinopathy positive, 71 retinopathy negative, 2 retinopathy unknown) for analysis by metagenomic next-generation sequencing. We found CSF viral coinfections in 7/38 (18.4\%) retinopathy-positive children and in 18/71 (25.4\%) retinopathy-negative children. Excluding HIV-1, human herpesviruses (HHV) represented $61 \%$ of viruses identified. Excluding HIV-1, CNS viral coinfection was equally likely in children who were retinopathy positive and retinopathy negative $(P=0.1431)$. Neither mortality nor neurological morbidity was associated with the presence of virus (odds ratio $[\mathrm{OR}]=0.276,95 \% \mathrm{Cl}$ : 0.056-1.363). Retinopathy-negative children with a higher temperature, lower white blood cell count, or being dehydrated were more likely to have viral coinfection. Level of consciousness at admission was not associated with CNS viral coinfection in retinopathy-negative children. Viral CNS coinfection is unlikely to contribute to coma in children with CM. The herpesviruses other than herpes simplex virus may represent incidental bystanders in CM, reactivating during acute malaria infection.
\end{abstract}

\section{INTRODUCTION}

Malaria continues to have a profound public health impact across Africa. Of the 500,000 people who develop severe malaria annually, 100,000 die and many survivors suffer physical and neurological sequelae. ${ }^{1-4}$ The most feared form of disease is cerebral malaria (CM), defined as coma with Plasmodium parasitemia and no other explanation for illness. ${ }^{1}$

Children with $\mathrm{CM}$ may be clinically grouped by the presence or absence of malarial retinopathy. ${ }^{5}$ Autopsies of children dying from retinopathy-positive CM reveal high levels of sequestration of parasitized erythrocytes in cerebral vasculature, considered CM's pathological hallmark. ${ }^{6}$ Postmortem studies of retinopathy-negative CM cases demonstrate lower amounts of sequestration in cerebral vasculature and evidence of non-malarial etiologies of coma and death, such as viral encephalitis. $^{6}$

Investigators studying retinopathy-negative CM have hypothesized that a large proportion of these children have asymptomatic malaria parasitemia (reflecting residence in an area of high malaria transmission) coupled with a non-malarial etiology of coma. ${ }^{7-9}$ Although autopsy studies confirm that nonmalarial etiologies of coma may be present in children dying of retinopathy-negative $\mathrm{CM},{ }^{6}$ the proportion of survivors with nonmalarial coma is unknown. Without a clear animal model of CM or malarial retinopathy, the etiology of illness in children with retinopathy-negative $\mathrm{CM}$ and survivable disease is unclear.

\footnotetext{
*Address correspondence to Douglas G. Postels, Department of Neurology, Children's National Medical Center, George Washington University, 111 Michigan Ave. NW, Washington, DC 20010. E-mail: dpostels@childrensnational.org
}

With fever, coma, and recurrent seizures, the presentation of children with viral encephalitis is similar to CM. Viral central nervous system (CNS) infection is common worldwide and, like $\mathrm{CM}$, results in high mortality and neurological morbidity in survivors. ${ }^{10}$ The possibility that retinopathy-negative CM may be driven in part by CNS viral coinfections could have significant management implications as adjunctive antiviral therapies targeting coinfections might reduce mortality or morbidity currently attributed to $\mathrm{CM}$.

To evaluate for this possibility, we collected clinical data and cerebrospinal fluid (CSF) specimens from children with retinopathy-negative and retinopathy-positive $\mathrm{CM}$. Our goals were to determine the proportion of children with retinopathynegative CM with CNS viral coinfections that may be contributing to illness, establish whether clinical parameters could differentiate children with retinopathy-negative CM who did and did not have a virus in the CNS, and determine whether the presence of a virus changed the mortality or morbidity risk. We also tested the hypothesis that identification of a CNS virus was more common in children with retinopathy-negative CM, compared with those who were retinopathy positive.

\section{MATERIALS AND METHODS}

From May 2014 to June 2015, we prospectively enrolled pediatric inpatients with a clinical diagnosis of CM at Queen Elizabeth Central Hospital (Blantyre, Malawi) and Komfo Anyoke Teaching Hospital (Kumasi, Ghana). Cerebral malaria was diagnosed using the following criteria: 1) coma (Blantyre coma scale score $\leq 2$ in children $\leq 5$ years and/or Glasgow Coma Scale score $\leq 8$ in children $>5$ years), 2) Plasmodium falciparum on blood smear, and 3) no other known cause of 
coma (e.g., meningitis, a prolonged postictal state or hypoglycemia-associated coma reversed by glucose infusion). Children with cloudy CSF (in Malawi and Ghana) or CSF white blood cell count of $>10 \mathrm{WBC} / \mu \mathrm{L}$ (in Uganda) were considered to have possible meningitis and excluded. Informed consent was obtained from parents or guardians. The study was reviewed and approved by the Institutional Review Boards of Michigan State University (USA), the College of Medicine Research Ethics Committee, University of Malawi (Blantyre, Malawi), and the Kwame Nkrumah University of Science and Technology (Kumasi, Ghana). Analysis of deidentified biological specimens was approved by the Institutional Review Board of Makerere University (Kampala, Uganda) and the University of California, San Francisco (USA).

To increase study power and geographical generalizability, we retrospectively collected and analyzed clinical data and CSF gathered from children admitted to the same Malawian hospital unit (admission dates from January 2008 to April 2014) and at Mulago Hospital (Kampala, Uganda, dates of admission from November 2008 to December 2013). We obtained informed consent from parents or guardians of all prospective enrollees. The parents of children retrospectively enrolled had consented to the analysis of de-identified archived biological specimens collected during prior observational research studies of malaria pathogenesis.

We identified malarial retinopathy at admission by direct and indirect ophthalmoscopy after bilateral pupillary dilation. A physician determined malarial retinopathy status in Ghana and Uganda, and an ophthalmologist had previously validated these physicians' identification of retinopathy. In Malawi, an ophthalmologist determined retinopathy status.

All children received the standard of care at their respective hospitals. This included intravenous antimalarials (quinine until 2008, artesunate thereafter in Malawi and Ghana; quinine for the full study period in Uganda), anticonvulsants for clinical seizures, careful fluid and nutrition management, and supportive care. Mechanical ventilation was unavailable.

After medical stabilization, we obtained CSF by routine lumbar puncture (LP) as soon as possible, typically within 1 hour of enrollment. After skin cleansing, a 21-gauge straight needle was introduced into the L3-4 interspace. Prospectively enrolled subjects had $3 \mathrm{~mL}$ of CSF withdrawn. Archived CSF from Uganda and subjects from Malawi admitted before May 2014 was of lower volume, often less than 500 microliters.

We analyzed CSF specimen with metagenomic nextgeneration sequencing (mNGS) of total RNA extracted from CSF, allowing unbiased interrogation of both known and novel viruses. Metagenomic sequencing library preparation and bioinformatic analyses were performed according to previously published protocols. ${ }^{11}$

Because of financial constraints, we did not analyze all collected CSF samples, but randomly selected CSF samples from Ghana and Uganda for analysis. In prospectively collected and archived Malawian samples, we preferentially analyzed 53 CSF samples collected from children who were retinopathy negative. We randomly selected 58 of the remaining 219 CSF specimens collected at all three sites for analysis; in total, $71(63.4 \%)$ of the analyzed samples were from retinopathy-negative children.

Although mNGS can identify neurologic infections from bacteria, viruses, fungi, and parasites, the samples' CSF cellular profiles were largely not consistent with a fulminant bacterial, fungal, or parasitic meningitis (all had CSF WBC counts $\leq 10 / \mu \mathrm{L}$ ), making the pretest probability low that these were relevant pathogens. In addition, there was a high burden of nonpathogenic bacterial and fungal sequences identified by mNGS in these CSF samples, likely as a result of environmental contamination, making it difficult to confidently identify nonviral infections.

Viral pathogens identified by mNGS were considered positive if specific criteria were met. Only alignments to viruses known to exist as human pathogens were considered. Alignments to these viruses were manually confirmed for accuracy in both the NCBI nucleotide and protein databases. Because herpes simplex virus (HSV) infections would be atypical for the study cohort's clinical phenotype, we required detection of HSV on two independent sequencing runs.

Statistical analysis. HIV-1 RNA was detectable by mNGS in the CSF of all HIV-1 seroreactive subjects and in none of the HIV-1 seronegative subjects. Because the role of HIV-1 in CNS disease is still incompletely understood, we analyzed viral data both with and without including HIV-1 as a potential CNS pathogen.

To determine whether clinical parameters could differentiate retinopathy-negative cases with or without a virus detected in the CNS, we used logistic regression. The candidate pool of potential variables included both admission clinical (age, temperature, pulse, blood pressure, respirations, capillary refill (in seconds), liver and spleen size, lymphadenopathy, jaundice, neck stiffness, and seizures) and laboratory findings (admission hematocrit, white blood cell count, platelets, glucose, quantitative $P$. falciparum histidine-rich protein 2 (a malaria parasite derived protein), HIV-1 serostatus, and CSF white blood cell count). We removed neck stiffness because of the rarity of this finding (1 of 111 , which caused quasiseparation). After excluding subjects with missing data, information from 50 (of 71) retinopathy-negative subjects was used to determine the predictive model.

Using a branch-and-bound algorithm, four models with the highest likelihood score (chi-square test) statistic for model sizes from 1 to 17 and a full model including all candidate variables were automatically selected by the best score selection method using SAS version 9.4 (SAS Corporation, Cary, $\mathrm{NC})$. To compare those models, Akaike information criterion (AIC) was used. The model with the smallest AIC score was chosen, allowing a more precise prediction. Because we analyzed data in two ways (including all viruses and excluding HIV-1 as a potential CNS pathogen), we created two predictive models, one for each analysis.

To determine whether the presence of a virus in the CSF increased mortality or morbidity risk in children with $\mathrm{CM}$, we used a logistic regression model in which we adjusted for known risk factors for death or neurological disability in this population, including admission glucose and coma score. To adjust for the potential confounding of retinopathy status and investigate the effects of viral coinfection in the subgroups of retinopathypositive and retinopathy-negative children, we performed another logistic regression which added the retinopathy status and its interaction with viral coinfection to the previous model.

To test the hypothesis that virus detection was more common in children with retinopathy-negative $\mathrm{CM}$, we compared proportions of virus-positive cases in retinopathy-negative versus retinopathy-positive patients using Pearson chi-square analyses or Fisher's exact test if the sample size was small. 
Retinopathy status was determined by ophthalmologists in Malawi and non-ophthalmologist physicians in Ghana and Uganda. Because determinations of retinopathy status may have been more accurate in Malawi, we performed sub-analyses, restricting data to that collected in Malawi. In all analyses, a $P$-value $<0.05$ was considered statistically significant.

\section{RESULTS}

We enrolled 272 subjects across the three sites. Enrollees in Ghana were mostly retinopathy negative; in Uganda and Malawi, most CSF samples were from children who were retinopathy positive (Table 1). After dividing the samples by country and type of enrollment, we selected CSF from 111 children for analysis: 38 retinopathy positive, 71 retinopathy negative, and two with retinopathy status unknown (Table 1). Comparing demographic and outcome features of children whose CSF was analyzed with those whose CSF was not, there was no significant difference in the age, gender, or rates of death or neurological sequelae in survivors (data not shown).

We found coinfection with a diversity of viruses (Figure 1). Twenty-five children (seven retinopathy positive) had viral sequences detected in the CSF, including four children in whom more than one virus was detected. Excluding HIV-1, herpesviruses were the predominant pathogens, accounting for $61.1 \%$ (11 of 18 ) of viruses. HSV-1, varicella zoster virus (VZV), and human herpes virus (HHV) eight were each detected once, Epstein-Barr virus (EBV) three times, and cytomegalovirus (CMV) four times. In addition to HIV-1 and HHVs, we identified hepatitis $B$ virus, hepatitis $A$ virus, polyomavirus 6 , and parvovirus B19.

Analyses including all viruses. We found viruses in 25 of the 111 (22.5\%) subjects with CM, 18.4\% (7/38) of those who were retinopathy positive and $25.4 \%(18 / 71)$ of those who were retinopathy negative (Figure 1). When comparing children who were retinopathy positive and retinopathy negative, there was no statistically significant difference in the proportion of CSF specimens with detected viruses $(P=0.4121)$.

All children who were HIV-1 seroreactive had detectable CSF HIV-1 nucleic acid. This led to quasi-separation in building the predictive model of CNS viral infection. We used Firth logistic regression to address this issue. The selected predictive model of viral infection includes HIV-1 serostatus, temperature, dehydration status, and platelet as predictors. Children with positive HIV-1 serostatus $(\mathrm{OR}=15.670,95 \% \mathrm{Cl}$ : 1.485-165.378), higher temperature (for each $1^{\circ} \mathrm{C}$ increase in body temperature, $\mathrm{OR}=3.164,95 \% \mathrm{Cl}$ : 1.417-7.067), lower platelet count (for $1 \times 10^{9}$ per liter decrease in platelet count, $\mathrm{OR}=1.007,95 \% \mathrm{Cl}: 0.999-1.014)$, or being dehydrated (OR = 18.346, 95\% Cl: 0.329-1,023.026) were more likely to have a virus detected. Depth of coma was not associated with the presence of a viral coinfection.

Without controlling for retinopathy status, the risk of an adverse outcome (death or neurological sequelae) was inversely associated with the presence of virus $(O R=0.244$, 95\% Cl: 0.064-0.930). Those with a viral coinfection had a lower risk of mortality or neurological morbidity compared with those who were not coinfected. When stratified by retinopathy status, neither mortality nor neurological morbidity was associated with viral detection (retinopathy negative: OR = 0.266, 95\% Cl: 0.067-1.062) (retinopathy positive: OR = $0.334,95 \% \mathrm{Cl}: 0.014-8.270)$.

In our sub-analyses limiting data to that collected in Malawi, our findings were similar. We found viruses in 20 of the 66 (30.3\%) subjects with CM from Malawi, 23.1\% (3/13) of those who were retinopathy positive and $32.1 \%(17 / 53)$ of those who were retinopathy negative (Figure 1). When comparing children who were retinopathy positive and retinopathy negative, there was no statistically significant difference in the proportion of CSF specimens with detected viruses (Fisher's exact test, $P=0.7395270$ ).

Without controlling for retinopathy status, the risk of an adverse outcome (death or neurological sequelae) was inversely associated with the presence of virus (OR $=0.122,95 \% \mathrm{Cl}$ : 0.021-0.692). Those with a viral coinfection had a lower risk of mortality or neurological morbidity compared with those who were not coinfected. When stratified by retinopathy status, the risk of an adverse outcome (death or neurological sequelae) was inversely associated with the presence of virus among the retinopathy-negative group, whereas not among the positive group (retinopathy negative: $\mathrm{OR}=0.150,95 \% \mathrm{Cl}: 0.028-0.792$ ) (retinopathy positive: $\mathrm{OR}=0.554,95 \% \mathrm{Cl}$ : 0.012-25.209).

Analyses including all viruses but excluding HIV-1 as a potential CNS pathogen. There were non-HIV-1 viruses in the CSF of 16 of the 111 (14.4\%) subjects with CM, 7.9\% $(3 / 38)$ of those who were retinopathy positive, and $18.3 \%$ $(13 / 71)$ of those who were retinopathy negative. Of the viruses detected, 11 of the $18(61.1 \%)$ were human herpesviruses. When comparing children who were retinopathy positive and retinopathy negative, there was no statistically significant difference in the proportion of CSF specimens with virus detected $(P=0.1431)$.

The selected predictive model of CNS viral infection includes temperature, dehydration status, and white blood cell count as predictors. Those with higher temperature (for each $1^{\circ} \mathrm{C}$ increase in body temperature, $\mathrm{OR}=1.851,95 \% \mathrm{Cl}$ : 0.953-3.595), lower white blood cell count (for $1 \times 10^{3}$ per $\mu \mathrm{L}$ decrease in white blood cell count, $\mathrm{OR}=1.202,95 \% \mathrm{Cl}$ : 1.000-1.445), or capillary refill time $>2$ seconds $(O R=31.128$, 95\% Cl: $0.559-1,733.367)$ were more likely to have a virus detected. Level of consciousness was not associated with CSF viral coinfection.

TABLE 1

Origin of cerebrospinal fluid samples collected and analyzed

\begin{tabular}{|c|c|c|c|c|c|c|}
\hline Site & $\begin{array}{l}\text { Prospective/retrospective } \\
\text { enrollment }\end{array}$ & Dates of sample collection & $\begin{array}{c}\text { Number of children } \\
\text { with samples available } \\
\text { for testing }\end{array}$ & $\begin{array}{l}\text { Percent of children who } \\
\text { were retinopathy } \\
\text { negative }\end{array}$ & $\begin{array}{c}\text { Samples analyzed by } \\
\text { metagenomic next- } \\
\text { generation sequencing }\end{array}$ & $\begin{array}{l}\text { Samples analyzed } \\
\text { from retinopathy- } \\
\text { negative subjects }\end{array}$ \\
\hline Ghana & Prospective & May 2014-March 2015 & 48 & $29 / 48(60.4 \%)$ & $17 / 48(35.4 \%)$ & $11 / 17(64.7 \%)$ \\
\hline Uganda & Retrospective & $\begin{array}{l}\text { November 2008- } \\
\text { December } 2013\end{array}$ & 33 & $8 / 33(24.2 \%)$ & $28 / 33(84.8 \%)$ & $7 / 28(25.0 \%)$ \\
\hline Malawi & Prospective and retrospective & January 2008-April 2015 & 191 (19 prospective) & $69 / 191(36.1 \%)$ & $66 / 191(34.6 \%)$ & $53 / 66(80.3 \%)$ \\
\hline
\end{tabular}



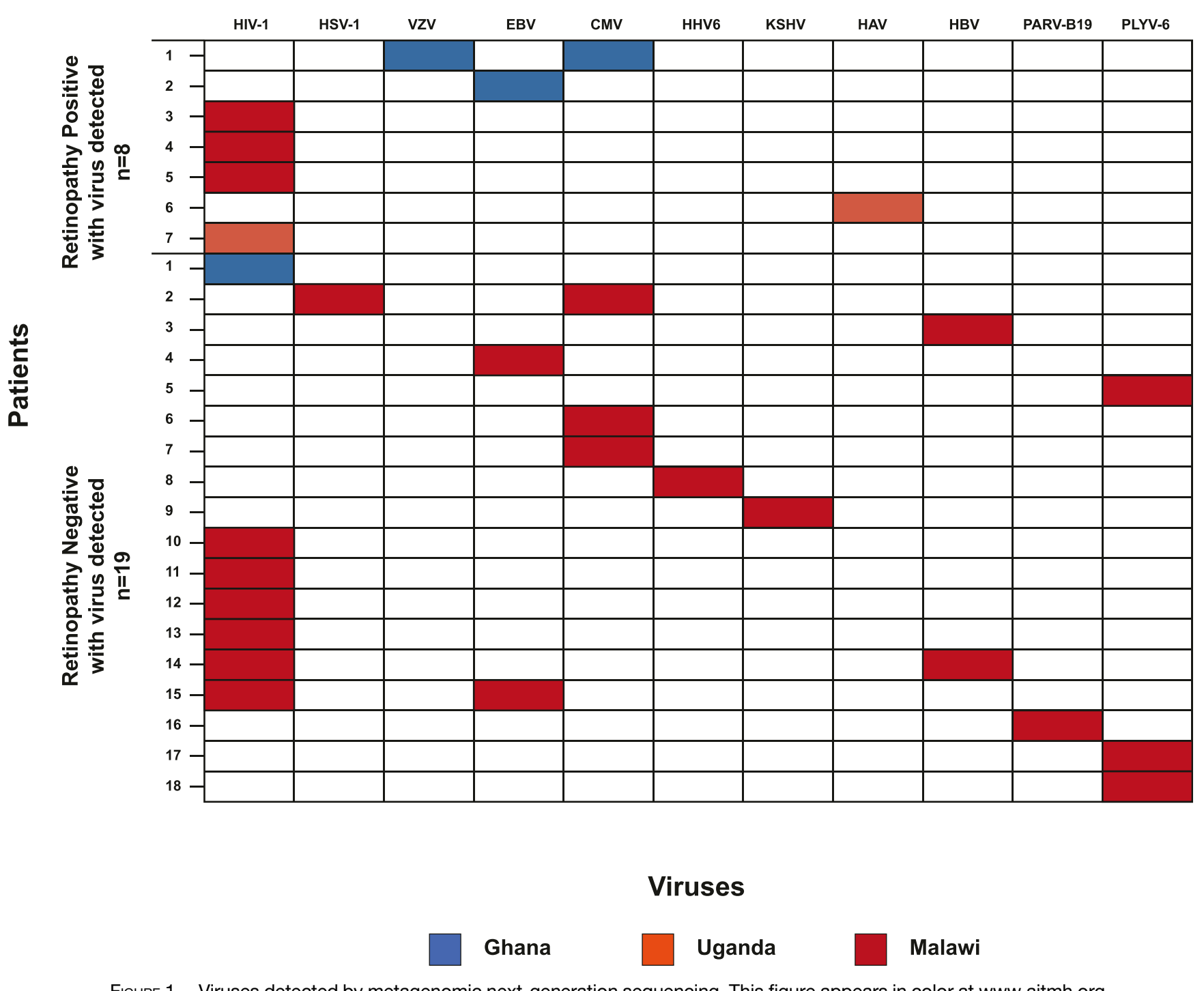

Without controlling for retinopathy status, the risk of mortality or neurological morbidity was not associated with the presence of virus $(\mathrm{OR}=0.276,95 \% \mathrm{Cl}$ : $0.056-1.363)$. When stratified by retinopathy status, neither mortality nor neurological morbidity was associated with viral detection (retinopathy negative: $\mathrm{OR}=0.283,95 \% \mathrm{Cl}: 0.059-1.372$ ) (retinopathy positive: $\mathrm{OR}=0.877,95 \% \mathrm{Cl}: 0.025-30.839$ ).

Restricting data from participants enrolled in Malawi, our findings were similar. We found viruses in 13 of the $66(19.7 \%)$ subjects with $\mathrm{CM}$ from Malawi, $0 \%(0 / 13)$ of those who were retinopathy positive, and $24.5 \%(13 / 53)$ of those who were retinopathy negative (Figure 1 ). When comparing children who were retinopathy positive and retinopathy negative, there was no statistically significant difference in the proportion of CSF specimens with detected viruses (Fisher's exact test, $P=$ 0.0562463).

Without controlling for retinopathy status, the risk of an adverse outcome (death or neurological sequelae) was not associated with the presence of virus $(\mathrm{OR}=0.286,95 \% \mathrm{Cl}$ : $0.051-1.595)$. Those with a viral coinfection had a lower risk of mortality or neurological morbidity compared with those who were not coinfected. Because virus was not detected in any retinopathy-positive subject, we could not add an interaction term between retinopathy and virus to the logistic regression model for the adverse outcome. Instead, we fit a logistic regression adding retinopathy as a covariate to the previous model, and another logistic regression to retinopathy-negative subjects only. After adjustment for retinopathy status, neither mortality nor neurological morbidity was associated with viral detection (OR $=0.232,95 \% \mathrm{Cl}$ : 0.041-1.320). In retinopathynegative subjects, neither mortality nor neurological morbidity was associated with viral detection (retinopathy negative: $\mathrm{OR}=0.24388,95 \% \mathrm{Cl}: 0.04357-1.363470)$.

\section{DISCUSSION}

Central nervous system viruses are common in the CSF of children with CM. Excluding HIV-1, their presence did not correlate with the outcome. Other than HIV-1, herpesviruses were most commonly identified, present in three-fourths of subjects with detectable viral sequences.

Many of the herpesviruses detected in our study are controversial in terms of their neurovirulence and ability to contribute to clinical disease. As such, it is, perhaps, unsurprising that their 
detection in the CNS of children with CM did not correlate with admission level of consciousness or outcome. Indeed, the presence of herpesvirus nucleic acid in the CSF may be due to primary CNS invasion or from reactivation of latent viruses during an acute illness; reactivation of herpesviruses has been described. ${ }^{12,13}$ HSV-1 and VZV cause CNS disease in the immunocompetent patients, but EBV, CMV, HHV-6, 7, and 8, are more likely to cause illness only in immunocompromised patients. Here, most patients with herpesvirus infection were HIV-1 negative, suggesting perhaps that their immune system was compromised by their general poor medical state (i.e., malnutrition) or their acute malaria infection.

A number of case reports for each of the non-HSV-1 or 2 herpesviruses suggest that they can cause encephalitis in immunocompetent patients, including reports highlighting HHV-6 and HHV-7 detected by PCR in children with suspected CNS infections. ${ }^{14,15}$ The similar rates of CSF herpesvirus detection in retinopathy-positive and retinopathy-negative children and lack of difference in mortality outcomes overall for children with and without non-HIV-1 viruses suggest that these infections are likely reactivations rather than newly acquired. Follow-up testing of cognition and neurologic function would have provided additional useful information about potential effects of coinfection but was not available at all study sites.

Similarly, although the significance of human parvovirus B19 detection in CSF is debated, infection of the CNS with this virus has been associated with encephalitis, including severe disease. ${ }^{16}$ Human polyoma virus-6 (HPyV6) detection in the CSF was an unexpected finding, occurring in three children, all with retinopathy-negative $\mathrm{CM}$. Human polyoma virus- 6 has been detected in the CSF of a patient with HIV-1 ${ }^{17}$, but its presence in the CSF is not otherwise reported. None of the three children with HPyV6 in the CSF had HIV-1 infection. The clinical relevance of this virus in CNS disease in immunocompetent children remains to be defined.

Our findings are congruent with those of Laman et al. ${ }^{18}$ who studied CNS viral infections in children with fever in Papua New Guinea. Children with febrile seizures or impairments of consciousness underwent diagnostic LP. Cerebrospinal fluid was analyzed by qPCR for HSV-1, HSV-2, VZV, HHV-5, HHV6 , picornaviruses, influenza $A$ and $B$, adenoviruses, flaviviruses, and bacteria (Streptococcus pneumoniae, HaemophiIus influenzae, and Neisseria meningitidis). Of the 300 children included, 69 had malaria parasitemia. Nineteen of these 69 (27.5\%) had CNS viral coinfections. HHVs accounted for $91 \%$ of the viral pathogens detected. A CNS viral coinfection did not change the risk of mortality or neurological morbidity in a child with malaria. Retinopathy status was not assessed.

In the largest previous study of CNS viral infections in Africa, Mallewa et al. ${ }^{19}$ collected CSF from children 2 months to 15 years old with suspected nonbacterial CNS infections. Five hundred thirteen children were recruited, and 164 (32\%) of the subjects had malaria parasitemia. Investigations for adenovirus, HSV-1, HSV-2, VZV, EBV, CMV, HHV-6, HHV-7, JC/BK viruses, VZV, rabies virus, and parvovirus $\mathrm{B} 19$, were performed on CSF by qualitative PCR. Forty-one (25\%) of the children with malaria parasitemia (both with normal and impaired consciousness) had a viral CNS coinfection. The most common pathogens detected were adenovirus (26.9\%), rabies (12.2\%), and HHV-6 (12.2\%). Of the 78 children with CM, $27(35.6 \%)$ had a CNS viral coinfection. The most common pathogen was adenovirus, found in $25.9 \%$ (7 or 27 ) of subjects. Including those with all forms of malaria, a viral coinfection significantly increased the mortality risk $(\mathrm{OR}=3.6$, 95\% Cl: 1.6-8.0). The authors did not analyze whether or not coinfection modified the outcome in the subgroup of children with $\mathrm{CM}$. In children with $\mathrm{CM}$, viral coinfection was not associated with malarial retinopathy.

In the current study, children with retinopathy-negative and retinopathy-positive $\mathrm{CM}$ were equally likely to have a viral coinfection. This suggests that this group of neuroinvasive viruses is not a differential contributor to illness in children with retinopathy-negative $\mathrm{CM}$. A recently published study revealed that the attributable fraction of disease in retinopathy-negative CM due to acute malaria infection is at least $85 \%$, supporting the hypothesis that acute malaria infection is the principal driver of illness in most patients with retinopathy-negative $\mathrm{CM}^{20}$ Comparison of clinical features ${ }^{21}$ and PfEMP1 expression $^{22}$ of children of differing retinopathy status similarly suggests that $P$. falciparum is the primary factor leading to disease in most children with retinopathy-negative CM. Although it is possible that some children with retinopathynegative $\mathrm{CM}$ may have asymptomatic parasitemia plus a non-malarial etiology of coma, our data do not support that this is commonly related to viruses.

Our study had advantages over previous investigations of CNS viral coinfection in CM. We gathered and analyzed specimens from three geographically distant sites in Africa. In addition, the use of mNGS allowed unbiased interrogation of CSF samples for viral pathogens, although we were unable to leverage the ability of mNGS to detect other infectious, nonviral causes of encephalitis given the bacterial and fungal environmental contamination in the samples.

This study has several limitations. We were unable to analyze concurrently collected serum for the presence of antibodies to any of the CNS viral pathogens detected and were unable to perform confirmatory qPCR on CSF to confirm the mNGS findings and determine the viral load. As the primary focus of our research was investigation of CNS viral detection in children with retinopathy-negative $\mathrm{CM}$, fewer children with retinopathy-positive $\mathrm{CM}$ were enrolled, limiting the study power for some analyses. We were unable to analyze all the CSF specimens collected, although we found no differences in demographics or outcomes between those whose specimens were analyzed, compared with those who were not. Determination of retinopathy status was performed by ophthalmologists in Malawi and by general physicians in Ghana and Uganda. Although ophthalmologists validated clinician's determinations of retinopathy status outside of Malawi, it is possible that retinopathy status assignments in Ghana and Uganda were less accurate, which could have led to misclassification. We conducted a sub-analysis limited to participants enrolled in Malawi and found similar results to the population included across the three countries.

Across the diverse geographic regions included, viral detection from CSF was not uncommon in children with CM. Human herpesviruses, which may undergo reactivation during acute malaria infection, ${ }^{23}$ were the predominant non-HIV-1 viruses found. Coupled with the facts that the presence of virus in CSF was unassociated with depth of coma at presentation and did not modify outcome, it is likely that herpesviruses are incidental bystanders in these children, becoming reactivated during acute malaria infection. 
Received December 23, 2019. Accepted for publication March 30, 2020.

Published online April 27, 2020.

Acknowledgments: We thank David Sambian for laboratory support in Ghana and Hedera Drury for aid with database management in Malawi.

Financial support: This work was supported by the Eunice Kennedy Shriver National Institute of Child Health \& Human Development of the National Institutes of Health under award number R21HD078471 (D. G. P.), the Clinical and Translational Sciences Institute of Michigan State University (D. G. P.), and the Sandler Foundation and William K. Bowes, Jr. Foundation (M. R. W., J. L. D., and L. M. K.). Additional funding was provided by the National Institutes of Health under award numbers R01NS055349 (C. C. J.), D43NS078280 (C. C. J.), K23HL138461-01A1 (C. L.), and K08NS096117 (M. R. W.).

Disclaimer: The content is solely the responsibility of the authors and does not necessarily represent the official views of the National Institutes of Health.

Authors' addresses: Douglas G. Postels, Department of Neurology, Children's National Medical Center, George Washington University, Washington, DC, E-mail: dpostels@childrensnational.org. Lawrence Osei-Tutu, Department of Child Health, Komfo Anokye Teaching Hospital, Kumasi, Ghana, E-mail: oseitutlaw@gmail.com. Karl B. Seydel and Terrie E. Taylor, Department of Osteopathic Medical Specialties, Michigan State University, East Lansing, Michigan, E-mails: seydel@msu.edu and ttmalawi@msu.edu. Qian Xu and Chenxi Li, Department of Epidemiology and Biostatistics, Michigan State University, East Lansing, MI, E-mails: xuqian3@msu.edu and cli@msu.edu. Chandy C. John, Ryan White Center for Pediatric Infectious Diseases and Global Health, Department of Pediatrics, Indiana University School of Medicine, Indianapolis, IN, E-mail: chjohn@ iu.edu. Macpherson Mallewa, University of Malawi College of Medicine, Blantyre, Malawi, E-mail: mmallewa@medcol.mw. Tom Solomon, Institute of Infection and Global Health, University of Liverpool, Liverpool, United Kingdom, E-mail: tsolomon@liverpool.ac.uk. Robert O. Opoka, Department of Paediatrics and Child Health, Makerere University School of Medicine, Kampala, Uganda, E-mail: opokabob@ yahoo.com. Tsiri Agbenyega and Daniel Ansong, Kwame Nkrumah University of Science and Technology, Kumasi, Ghana, E-mails: tsiri@ ghana.com and ansongd@yahoo.com. Lillian M. Khan and Joseph L. DeRisi, Department of Biochemistry and Biophysics, University of California San Francisco, San Francisco, CA, E-mails: lillianmkhan@ gmail.com and joe@derisilab.ucsf.edu. Kristoffer E. Leon, UCSF School of Medicine, University of California San Francisco, San Francisco, CA, E-mail: kristoffer.leon@ucsf.edu. Charles Langelier, Division of Infectious Diseases, Department of Medicine, University of California San Francisco, San Francisco, CA, E-mail: chaz.langelier@ ucsf.edu. Michael R. Wilson and Prashanth S. Ramachandran, UCSF Weill Institute for Neurosciences, San Francisco, CA and Department of Neurology, University of California San Francisco, San Francisco, CA, E-mails: michael.wilson@ucsf.edu and prashanth.ramachandran@ ucsf.edu.

\section{REFERENCES}

1. 2014. Severe malaria. Trop Med Int Health 19 (Suppl 1): 7-131.

2. WHO, 2014. WHO World Malaria Report. Geneva, Switzerland: World Health Organization.

3. Birbeck GL, Molyneux ME, Kaplan PW, Seydel KB, Chimalizeni YF, Kawaza K, Taylor TE, 2010. Blantyre malaria project epilepsy study (BMPES) of neurological outcomes in retinopathypositive paediatric cerebral malaria survivors: a prospective cohort study. Lancet Neurol 9: 1173-1181.
4. Postels DG, Taylor TE, Molyneux M, Mannor K, Kaplan PW, Seydel KB, Chimalizeni YF, Kawaza K, Birbeck GL, 2012. Neurologic outcomes in retinopathy-negative cerebral malaria survivors. Neurology 79: 1268-1272.

5. Lewallen S, Taylor TE, Molyneux ME, Wills BA, Courtright P, 1993. Ocular fundus findings in Malawian children with cerebral malaria. Ophthalmology 100: 857-861.

6. Taylor TE, Fu WJ, Carr RA, Whitten RO, Mueller JS, Fosiko NG, Lewallen S, Liomba NG, Molyneux ME, 2004. Differentiating the pathologies of cerebral malaria by postmortem parasite counts. Nat Med 10: 143-145.

7. Potchen MJ, Birbeck GL, Demarco JK, Kampondeni SD, Beare N, Molyneux ME, Taylor TE, 2010. Neuroimaging findings in children with retinopathy-confirmed cerebral malaria. Eur J Radiol 74: 262-268.

8. Seydel KB et al., 2015. Brain swelling and death in children with cerebral malaria. N Engl J Med 372: 1126-1137.

9. Taylor TE, Molyneux ME, 2015. The pathogenesis of pediatric cerebral malaria: eye exams, autopsies, and neuroimaging. Ann NY Acad Sci 1342: 44-52.

10. Munoz LS, Garcia MA, Gordon-Lipkin E, Parra B, Pardo CA, 2018. Emerging viral infections and their impact on the global burden of neurological disease. Semin Neurol 38: 163-175.

11. Wilson MR et al., 2018. Chronic meningitis investigated via metagenomic next-generation sequencing. JAMA Neurol 75: 947-955.

12. Agut $H$, Bonnafous $P$, Gautheret-Dejean $A, 2015$. Laboratory and clinical aspects of human herpesvirus 6 infections. Clin Microbiol Rev 28: 313-335.

13. Traylen CM, Patel HR, Fondaw W, Mahatme S, Williams JF, Walker LR, Dyson OF, Arce S, Akula SM, 2011. Virus reactivation: a panoramic view in human infections. Future Virol 6: 451-463.

14. Ongrádi J, Ablashi DV, Yoshikawa T, Stercz B, Ogata M, 2017. Roseolovirus-associated encephalitis in immunocompetent and immunocompromised individuals. J Neurovirol 23: 1-19.

15. Yoshinari S, Hamano S, Minamitani M, Tanaka M, Eto Y, 2007. Human herpesvirus 6 encephalopathy predominantly affecting the frontal lobes. Pediatr Neurol 36: 13-16.

16. Watanabe $\mathrm{T}$, Kawashima $\mathrm{H}, 2015$. Acute encephalitis and encephalopathy associated with human parvovirus B19 infection in children. World J Clin Pediatr 4: 126-134.

17. Delbue S, Tremolada S, Branchetti E, Elia F, Gualco E, Marchioni $E$, Maserati R, Ferrante P, 2008. First identification and molecular characterization of lymphotropic polyomavirus in peripheral blood from patients with leukoencephalopathies. J Clin Microbiol 46: 2461-2462.

18. Laman M et al., 2014. Viral pathogens in children hospitalized with features of central nervous system infection in a malariaendemic region of Papua New Guinea. BMC Infect Dis 14: 630.

19. Mallewa $\mathrm{M}$ et al., Viral CNS infections in children from a malariaendemic area of Malawi: a prospective cohort study. Lancet Glob Health 1: e153-e160.

20. Small DS, Taylor TE, Postels DG, Beare NA, Cheng J, MacCormick IJ, Seydel KB, 2017. Evidence from a natural experiment that malaria parasitemia is pathogenic in retinopathy-negative cerebral malaria. Elife 6: e23699.

21. Villaverde C, Namazzi R, Shabani E, Opoka RO, John CC, 2017. Clinical Comparison of retinopathy-positive and retinopathynegative cerebral malaria. Am J Trop Med Hyg 96: 1176-1184.

22. Shabani E, Hanisch B, Opoka RO, Lavstsen T, John CC, 2017. Plasmodium falciparum EPCR-binding PfEMP1 expression increases with malaria disease severity and is elevated in retinopathy negative cerebral malaria. BMC Med 15: 183.

23. Chene A, Nylen S, Donati D, Bejarano MT, Kironde F, Wahlgren M, Falk KI, 2011. Effect of acute Plasmodium falciparum malaria on reactivation and shedding of the eight human herpes viruses. PLoS One 6: e26266. 